\title{
An Optimum Cost Handover Algorithm for LEO Satellites
}

\author{
Debabrata sarddar \\ (Asst Professor) \\ Department of C.S.E. \\ University of kalyani
Suman Kumar Sikdar
M. Tech in C.S.E
Jadavpur University

\author{
Soumya Das \\ Department of E.T.C.E. \\ Bengal Institute of tec.WBUT
}

\author{
Sougata Chakraborty \\ M.Tech in C.S.E \\ Jadavpur University
}

\author{
Dipsikha Ganguly \\ Department of E.T.C.E \\ B.P.Poddar I.M.T.,WBUT
}

\author{
Kunal Hui \\ M. Tech in C.S.E \\ Haldia Institute of Technology
}

\author{
Sujoy Palit \\ M. Tech in C.S.E \\ W.B.U.T
}

\begin{abstract}
Low Earth Orbit (LEO) satellite networks are foreseen to complement terrestrial networks in future communication. LEO satellites have important advantages such that low endto-end delay and power requirement and more efficient frequency spectrum allocation due to frequency reuse between satellite and spot beams. But the speed of LEO satellites is much higher than mobile nodes. As a result the handover occurrence is more. Handover may degrade system performance depending upon techniques employed by the satellite systems. So an appropriate handover scheme must be devised to increase system performance. This paper introduces a new handover algorithm which optimizes between the cost of handover and quality of service (QOS) parameters. Also it decreases handover delay. Simulations indicates that this algorithm is better than current algorithms in shorten transmission delay and minimizing handover cost.
\end{abstract}

\section{Keywords:}

Satellite handover; QOS; LEO satellite:Mobile Node (MN).

\section{Introduction:}

In recent years, extensive researches are investigated in order to provide user global network coverage anytime and anywhere. Modern terrestrial wireless networks such as mobile networks provide communication to a limited geographical area. In order to provide a global coverage satellite constellation system seem to be a promising choice. So the application of satellite networks extends from traditional telephone and TV broadcast service to data service such as file downloading and uploading and internet browsing.

There are mainly four types of satellite communication system exists depending upon the types of satellites

i) Geostationary Satellite (GEO)

ii) Medium orbit satellite (MEO)

iii) Low earth orbit satellite (LEO) although mixed constellations exists.

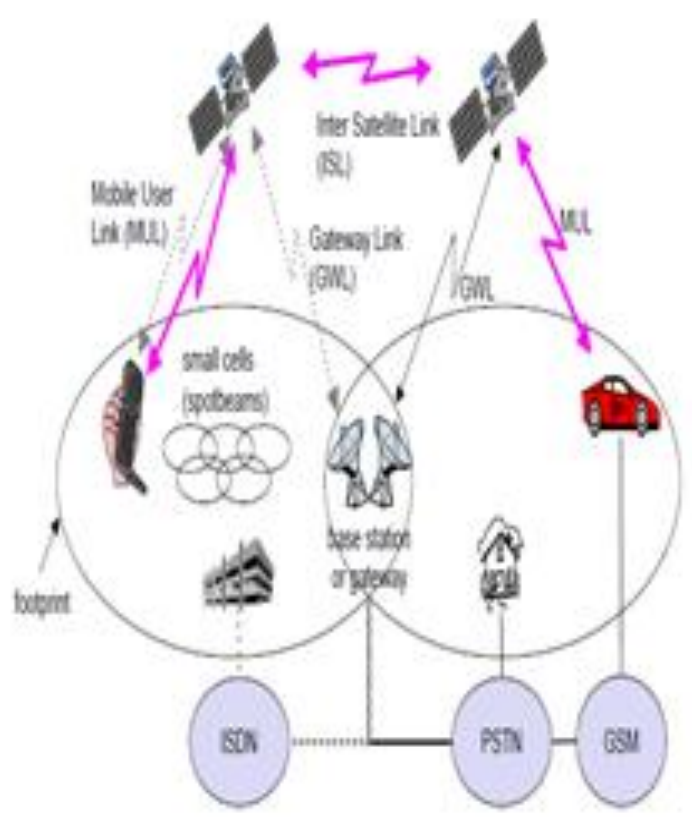

Fig.1 Satellite communication architecture

Comparing with other systems, LEO satellite systems is most preferable because of its different advantages such as low propagation delay, low handoff latency, low power requirement and effective bandwidth utilization.

But is some disadvantage also. The main disadvantage is the speed of the satellite is very high than MN's and speed of Earth. So the handover occurrence is more and the system design becomes more complex.[1]

\section{LOW-EARTH ORBIT (LEO)}

LEO systems fly about 1,000 kilometers above the Earth (between 400 miles and 1,600 miles) and, unlike GEOs, travel across the sky. A typical LEO satellite takes less than two hours to orbit the Earth, which means that a single satellite is "in view" of ground equipment for a only a few minutes. As a consequence, if a transmission takes more than the few minutes that any one satellite is in view, a LEO system must "hand off" between satellites in order to complete the transmission. In general, this can be accomplished by 
constantly relaying signals between the satellite and various ground stations, or by communicating between the satellites themselves using "inter-satellite links."

In addition, LEO systems are designed to have more than one satellite in view from any spot on Earth at any given time, minimizing the possibility that the network will lose the transmission. Because of the fast-flying satellites, LEO systems must incorporate sophisticated tracking and switching equipment to maintain consistent service coverage. The need for complex tracking schemes is minimized, but not obviated, in LEO systems designed to handle only short-burst transmissions. [2]

\section{Benefits of the LEO conce}

- LEOs can offer a communications infrastructure to areas where there is insufficient population to justify a terrestrial based cellular network. This not only includes many developing countries but $80 \%$ of the US as well. This explains why most of the initiatives for LEOs have come from the USA.

- Many developing countries are interested in LEO systems as an alternative to investing in a very expensive terrestrial telecommunications infrastructure.

- Communication via LEOs does not suffer from the objectionably long transmission delays associated with geosynchronous systems.

- User equipment does not require high-power transmitters or highly directional antennae that need to be continually pointed to the satellite. In practice, transmit powers can be much lower than 1 watt.

- LEO satellites are technically much simpler and more robust than geo-synchronous satellites and are less likely to suffer catastrophic failure during deployment or during the satellite lifetime. [3]

Scanning: When a mobile station is moving away from its current satellite, it initiates the handoff process when the received signal strength and signal-to-noise-ratio have decreased below the threshold level. The MN now begins the scanning to find new satellite. It can either opt for a passive scan (where it listens for beacon frames periodically sent out by satellites) or chose a faster active scanning mechanism wherein it regularly sends out probe request frames and waits for responses for $\mathrm{T}_{\mathrm{MIN}}$ (min Channel Time) and continues scanning until $\mathrm{T}_{\mathrm{MAX}}$ (max Channel Time) if at least one response has been heard within $\mathrm{T}_{\mathrm{MIN}}$. Thus, $\mathrm{n} * \mathrm{~T}_{\mathrm{MIN}} \leq$ time to scan $n$ channels $\leq n^{*} T_{\text {MAX }}$. The information gathered is then processed so that the MN can decide which Satellite to join next. The total time required until this point constitutes $90 \%$ of the handoff delay.

Authentication: Authentication is necessary to associate the link with the new satellite. Authentication must either immediately proceed to association or must immediately follow a channel scan cycle. In pre-authentication schemes, the $\mathrm{MN}$ authenticates with the new satellite immediately after the scan cycle finishes.

Re-Association: Re-association is a process for transferring associations from old satellite to new one. Once the MN has been authenticated with the new satellite, re-association can be started. Previous works has shown re-association delay to be around 1-2 ms. The range of scanning delay is given by:$\mathrm{N} \times$ Tmin _ Tscan_ $\mathrm{N} \times$ Tmax

Where $\mathrm{N}$ is the total number of channels according to the spectrum released by a country, Tmin is Min Channel Time, Tscan is the total measured scanning delay, and Tmax is Max Channel Time[4]-[9].
Handovers may degrade the system performance as an unsuccessful handover results call blocking and forced call termination. From the user point of view forced call termination is less desirable than a new call blocking though both affect the performance of the system [12].

In this paper we have proposed a new handover algorithm called Optimum Cost Handover Algorithm (OCHO).

The paper is organized as follow: in the second section we have described the related handover algorithms available. In the third section we have described the details of OCHO algorithm. In the forth section the simulation results of related handover algorithms and $\mathrm{OCHO}$ algorithm based in handover cost and average handover delay. In the next section we conclude the whole paper and finally a future work is mention regarding this paper in section six.

\section{Related work:}

In recent times, Many Researchers paid their attention to solve this handover problem in a various way. They have found that the communication link of a path of any call will be broken down after handover. Thus the QOS requirements of ongoing service would be destroyed because of excessive handover [13].

Some researches shows that one could expect that a call experiences a handover request every one minute, which is due to the high speed movement of satellites, nearly $7 \mathrm{~km} / \mathrm{s}$. In the iridium satellite constellation, the average no of handover request per call attempt is on average equal to 5 [15].

Researchers showed that there mainly are two things happen after a call experiences a handover.

- $\quad$ First one is complete rerouting of the previous communication path. In this case a future communications proceed in a new route. The advantage of this one is that the optimal path is selected but it cause signaling overhead and excessive route establishment delay. This might cause handover call blocking and thus the system performance degrades.

- The second one is partial rerouting instead of complete reroute. This technique is simple in implementing and causing less overhead in the network compared with the first one. In this case the route is non-optimal and might cause inefficient utilization of the satellite resources.

The Footprint Handover Reroute Protocol (FHRP) [16] uses both complete rerouting and partial rerouting equally. In this case, when an ongoing call needs handover, it will search for a new path quickly by augmenting the existing route to guarantee less handover delay. This path is non-optimal one so after some time intervals it will update this non-optimal augmented route to an optimal one. Although in FHRP the optimal path is used for future communication but it has some disadvantages.

$>\quad$ In practical, it is difficult for FHRP to select update interval as it might results excessive non-optimal paths.

$>\quad$ Also FHRP ignores the requirements of ensuring the QOS being handed over.

Another study admitted that the frequency of routing can influence the network preference, but it did not indicate the optimal routing condition [18].

Zhang Tao et al proposes a satellite networks minimum cost handover (SMCH) algorithm [19], which is capable of guaranteeing the QOS of the handover and minimizing the operational costs. SMCH consists of two phrases route augmentation and rerouting. Furthermore, the algorithm attaches importance to whether the rerouting is needed after 
handover. As a determinant of the rerouting, the handover cost comprises two parts: the excessive cost of non-optimal path after route augmentation and the signaling cost after rerouting. Stresses are laid on minimizing the excessive cost after satellite handover.

1) Initialization of handover: The satellite $S$ sends a service handover request message including the current routing $U 1$ to the satellite $\mathrm{S} 1$.

2) Route augmentation: When the satellite $\mathrm{S} 1$ receives a message from the satellite $S$, the route augmentation will be run to obtain a new path from the satellite S1 to the terminal B quickly. If this phase ends up in success, the instantly established service connection would send a successful message to the gateway station to initiate the rerouting phase. Otherwise, an unsuccessful message would also be done.

3) Rerouting: When the gateway station receives a successful or an unsuccessful message about route augmentation from the satellite $\mathrm{S} 1$, the station first checks whether or not the rerouting is necessary. If it is, the original routing algorithm is performed. In this case, the key problem is whether to trigger the rerouting or not.

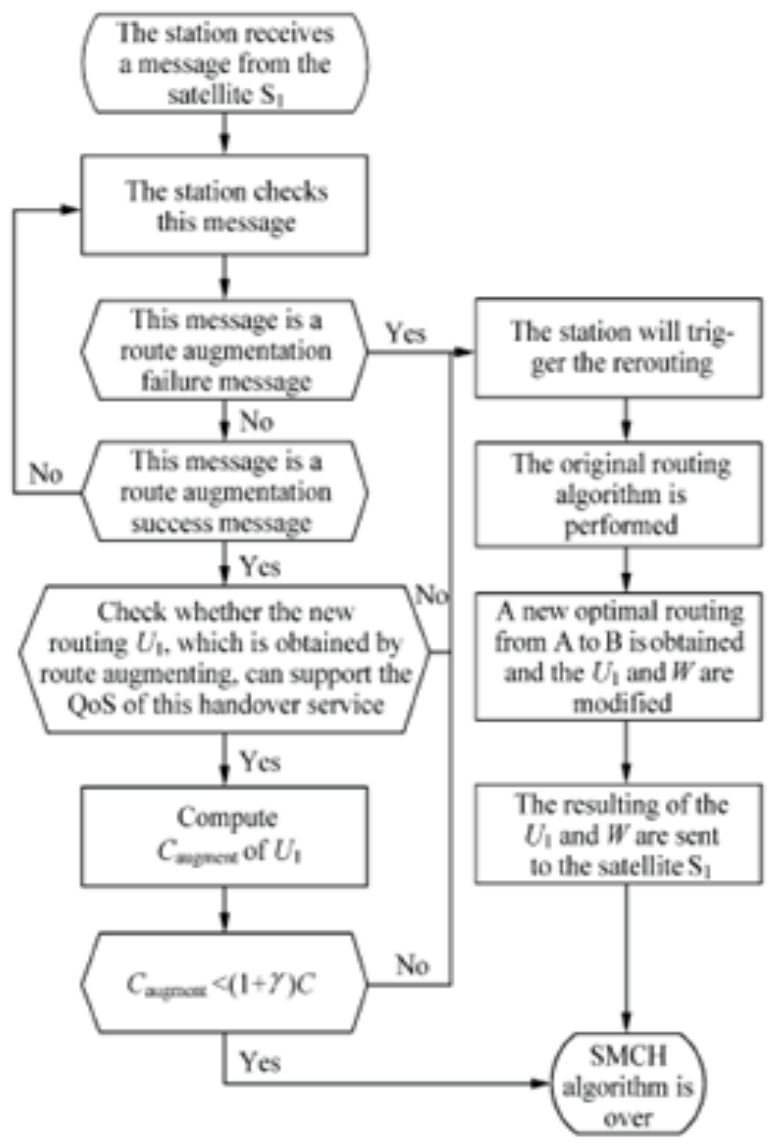

Fig.2 SMCH algorithm

The SMCH algorithm has three steps.

The details of SMCH algorithm is given in fig.2

Though SMCH algorithm is better than FHRP algorithm still it has following disadvantages.
I. In $\mathrm{SMCH}$ algorithm route augmentation is done first after handover and then, decides whether or not to update the route by rerouting. Now if the rerouting is done then we have to pay an additional cost of routing augmentation. Though this cost is much smaller but as the average no of handover is very high so we cannot ignore this cost. The details of cost analysis are given later in this article.

II. If rerouting is needed then the communication is done in two paths: first is the routing augmented path and then it follows the rerouting path. In this intermediate step the quality of signaling goes very low. As rerouting is very frequent so this degrades overall call quality. Sometimes the call can also be terminated in this intermediate step.

\section{Proposed Work:}

We have proposed a new handover algorithm termed as Optimum Cost Handover Algorithm (OCHO) where we have removed the extra cost of SMCH algorithm by minimising the cost and optimising the route.

OCHO algorithm is based on following facts

A. As the route of all the satellites is fixed so in a particular time of a day the positions of all the satellites are fixed. So we save this data then in any time the position of any satellite can be found easily.

B. The distance between the satellites in a particular time is also fixed. Once we have calculated these distances so we can find the signalling cost between any satellites in a particular time.

We define three levels for handover initialization according to the received signal strength $(\mathrm{SS})$ and signal-to-noise $(\mathrm{S} / \mathrm{N})$ ratio.

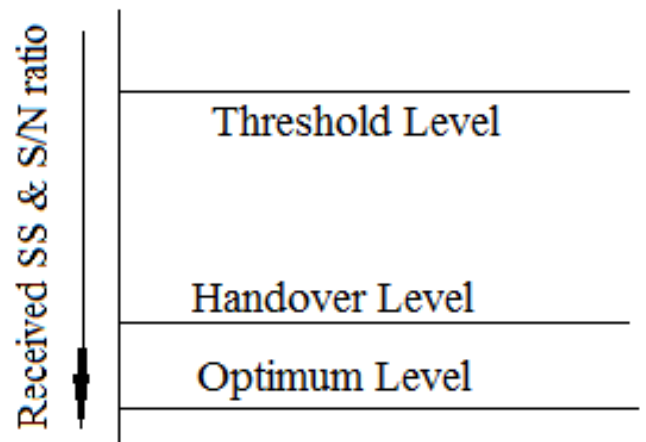

\section{Fig:3 Different handover levels}

A. $\quad$ Threshold level - When received SS and S/N this level the OCHO algorithm starts but the call will not be terminated. The choice of threshold level should me made very carefully.

B. Optimum level -When the received SS and S/N crosses this level the call will be terminated. The difference between optimum level and threshold level is equal to the time taken to complete the handover before the SS and S/N crosses the optimum level.

C. Handover level -When the received SS and S/N crosses this level the OCHO algorithm stops and the handover should be completed immediately. The difference between optimum level and Handover level is equal to the time taken to complete the handover before the SS and $\mathrm{S} / \mathrm{N}$ crosses the optimum level. Until the SS and S/N crosses this level the OCHO algorithm can continue but whenever SS and $\mathrm{S} / \mathrm{N}$ crosses this level we have to complete handover process 
otherwise it will cross the optimum level and the call will be terminated.

The significance of these three levels is that before every handover occurrence the path of signalling after handover is already known.

This algorithm can be implemented in ground station as well as in satellite node.

If the communication with mobile node is done directly with the satellites then this algorithm performed by the terminal satellite and the result is sent to the terminal satellite of the resulting path.

If the communication is done with mobile node to satellite by the ground base stations then this algorithm is performed by the ground stations.

Actually this algorithm creates a virtual environment of the path to be followed after algorithm. Different paths are obtained by this algorithm and we compare these paths to obtain the optimal path where the communication cost is minimised and also which satisfy the QOS of handover service. This virtual environment can be created by the data which are already saved in the database. This algorithm first calculated the total distance of any path from the knowledge of the positions of the satellite. Then it finds the cost of communication for that path. The algorithm is described as follows:

\section{OCHO Algorithm}

\section{OCHO algorithm has two parts}

I. Handover Preparation: The optimum path for signalling is selected by OCHO algorithm.

II. Handover Completion: The handover is completed by using the path selected by OCHO algorithm.

Let us assume that the communication with mobile node to satellite is done directly. Also we assume that there is a mobile node A connected with satellite $\mathrm{S} 1$ and the mobile node B is connected with satellite S3. In general the handover occurs in both the mobile node. Here we consider the handover in mobile node A. At the other terminal the same thing happens.

INITIAL SET UP: All the positions and their intersatellite distances and their corresponding signalling costs are saved in all satellites database. All the satellites use this database during handover.

STEP 1: The satellite S1 continuously checks if the received $\mathrm{SS}$ and $\mathrm{S} / \mathrm{N}$ is less than the Threshold level. If yes it performs the OCHO algorithm otherwise again it checks.

STEP 2: If the received SS and $\mathrm{S} / \mathrm{N}$ crosses the threshold level of signalling then the two operations are performed simultaneously. It also sets Cmin $=0, D \min =0, n=0$.

Using the database satellite $\mathrm{S} 1$ finds the nearest satellite to MN A at the direction of MN B. Suppose it finds satellite S4. Now it calculates if the signalling is done using the satellite S4 by routing augmentation then can it satisfy the QOS of handover service. If yes then it enters the STEP 2.A.a). else it performs STEP 2.B

Now using the database it calculates the total distance of signalling and from there it calculates the total cost $\mathrm{Ca}$ for this path.

Again using the database satellite $\mathrm{S} 1$ finds shortest distance to S3 for signalling $D(n)$ and set $\operatorname{Dmin}=D(n)$. After that it checks if the path Dmin can satisfy the QOS of handover service. If yes then it goes to STEP 3 else it goes to STEP 6.

STEP 3: Using the database it this step calculates the cost of signalling $\mathrm{C}_{\mathrm{r}}(\mathrm{n})$ for the path $\mathrm{D}_{\min }$.
STEP 4: This step checks if $\mathrm{C}_{\mathrm{a}}<\mathrm{C}_{\mathrm{r}}(\mathrm{n})$ ?. If yes then it sets $\mathrm{C}_{\min }=\mathrm{C}_{\mathrm{a}}$. Else it sets $\mathrm{C}_{\min }=\mathrm{C}_{\mathrm{r}}(\mathrm{n})$.

STEP 5: Now the received SS and S/N is compared with the Handover level. If it crosses the handover level then go to step 13, else go to step 6.

STEP 6: Set $\mathrm{n}=\mathrm{n}+1$.

$\underline{\text { STEP 7: }}$ Using the database again satellite $\mathrm{S} 1$ calculates another minimum distance $\mathrm{D}(\mathrm{n})$ such that $\mathrm{D}(\mathrm{n})>\mathrm{D}_{\text {min }}$ STEP 8: Set $\mathrm{D}_{\min }=\mathrm{D}(\mathrm{n})$.

STEP 9: Now check if the path Dmin can satisfy the QOS of handover service? If yes then go to step 10 else go to step 6.

STEP 10: Using the database calculate the cost of signalling $\mathrm{C}_{\mathrm{r}}(\mathrm{n})$ using the path $\mathrm{D}_{\min }$.

STEP 11: Compare if $\mathrm{C}_{\min }<\mathrm{C}_{\mathrm{r}}(\mathrm{n})$ ? If yes then go to step 5 else go to step 12.

STEP 12: Set $\mathrm{C}_{\min }=\mathrm{C}_{\mathrm{r}}(\mathrm{n})$ and go to step 5 .

STEP 13: Now as the received SS and $\mathrm{S} / \mathrm{N}$ crosses the handover level so the handover should be completed by the path whose cost is Cmin before it crosses the optimum level. The path obtained by the OCHO algorithm is sent to the satellite S4. Now satellite S4 finds the positions of the satellites in the resulting path using the database. Now it makes a connection set up for this path and the communication between $\mathrm{MN} \mathrm{A}$ and $\mathrm{MN} \mathrm{B}$ is done by the new path.

Before every handover any of the following three cases arises:

Case 1: The path obtained by routing augmentation is the optimal one.

Case 2: The path obtained by the direct link i.e., the minimum distance between the terminal satellites is the optimal path.

Case 3: The path obtained by the case 1 and case 2 is not the optimal path. So we have to find another path which optimises the cost and also satisfy QOS of service.

Now we will show how these three cases are implemented in our algorithm.

Case 1 is implemented in figure 4 Suppose at any time the position of satellite S1-S8 is shown in the figure. The communication between MN A and MN B is done by the path $\mathrm{A} \rightarrow \mathrm{S} 1 \rightarrow \mathrm{S} 2 \rightarrow \mathrm{S} 3 \rightarrow \mathrm{B}$. Now when the received $\mathrm{SS}$ and $\mathrm{S} / \mathrm{N}$ is below the threshold level, the satellite $\mathrm{S} 1$ performs OCHO algorithm. It first finds the path 


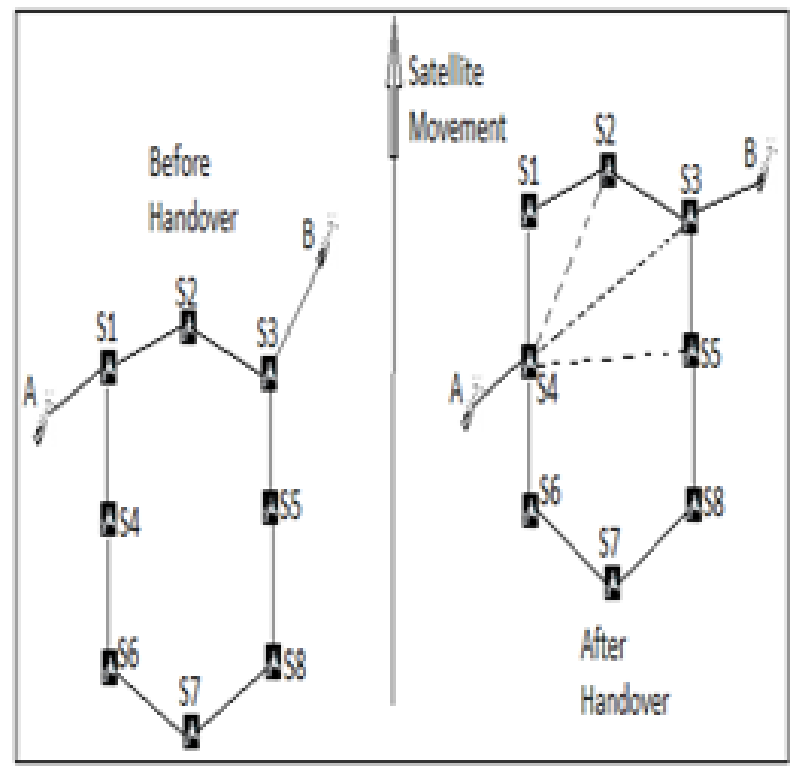

Fig.4 Case 1

$\mathrm{A} \rightarrow \mathrm{S} 4 \rightarrow \mathrm{S} 1 \rightarrow \mathrm{S} 2 \rightarrow \mathrm{S} 3 \rightarrow \mathrm{B}$. Simultaneously finds the direct link between $\mathrm{S} 4$ and $\mathrm{S} 3$ which is rejected as it does not satisfy the QOS of parameters as the inter-satellite distance is large. Similarly the path $\mathrm{A} \rightarrow \mathrm{S} 4 \rightarrow \mathrm{S} 2 \rightarrow \mathrm{S} 3 \rightarrow \mathrm{B}$ and $\mathrm{A} \rightarrow \mathrm{S} 4 \rightarrow \mathrm{S} 5 \rightarrow \mathrm{S} 3 \rightarrow \mathrm{B}$ is also rejected as it also does not satisfy the QOS of handover service. So we left only the route augmented path which satisfy the QOS of parameters and optimises the cost

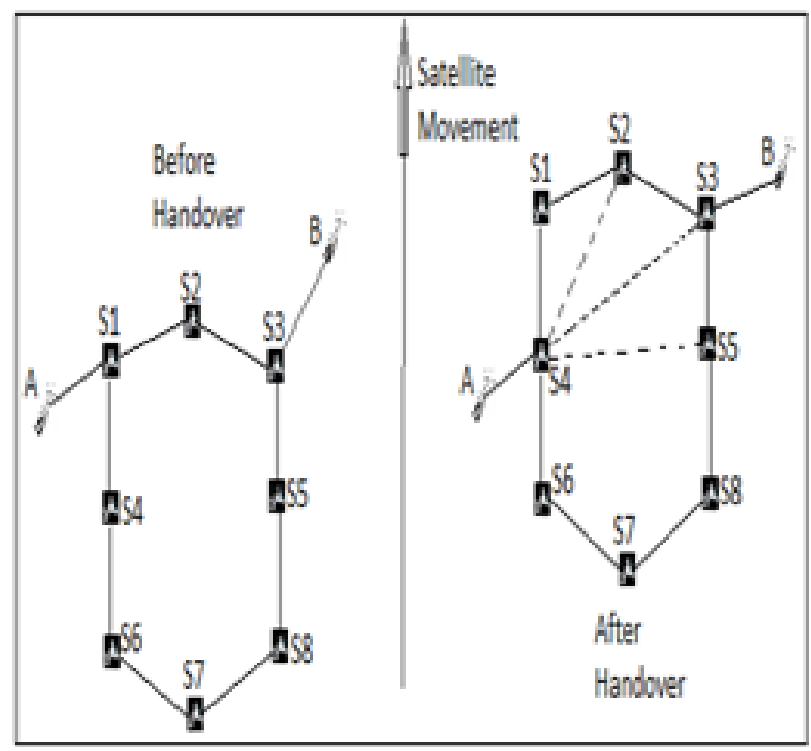

Fig.5 Case 2

Case 2 is implemented in figure 5. Here the scenario is different. Here first we find the route augmented path $\mathrm{A} \rightarrow \mathrm{S} 4 \rightarrow \mathrm{S} 1 \rightarrow \mathrm{S} 2 \rightarrow \mathrm{S} 3 \rightarrow \mathrm{B}$ which satisfies QOS of handover service. The direct link between MN A and MN B is the path $\mathrm{A} \rightarrow \mathrm{S} 4 \rightarrow \mathrm{S} 5 \rightarrow \mathrm{S} 6 \rightarrow \mathrm{B}$. But is satisfies the QOS of handover service. Also the cost $\mathrm{C}_{\mathrm{r}}(\mathrm{n}=0)$ is lower than the cost of route augmentation $\mathrm{Ca}$. We also find that this path is the optimal one among the other paths. So the communication after handover will be done in this path.

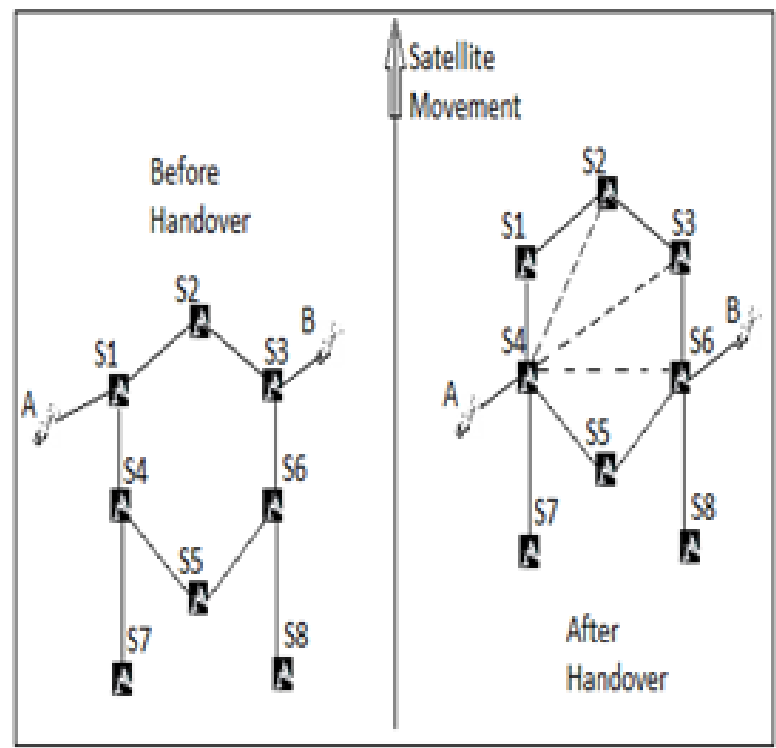

Fig.6 Case 3

Case 3 is shown in fig 6 . Here the route augmented path is $\mathrm{A} \rightarrow \mathrm{S} 4 \rightarrow \mathrm{S} 1 \rightarrow \mathrm{S} 2 \rightarrow \mathrm{S} 3 \rightarrow \mathrm{B}$. The direct linked path $\mathrm{A} \rightarrow \mathrm{S} 4 \rightarrow \mathrm{S} 6 \rightarrow \mathrm{B}$ is rejected as it does not satisfy the QOS of parameters. But the Path $\mathrm{A} \rightarrow \mathrm{S} 4 \rightarrow \mathrm{S} 5 \rightarrow \mathrm{S} 6 \rightarrow \mathrm{B}$ satisfies the QOS of handover service. Its cost $\mathrm{C}_{\mathrm{r}}(\mathrm{n}=1)$ is also less than $\mathrm{C}_{\mathrm{a}}$. The other paths are rejected as they either does not satisfy the QOS of handover service or their cost is higher than $\mathrm{C}_{\mathrm{r}}(\mathrm{n}=1)$ So in this case the communication is carried through this path.

\section{Cost Analysis:}

Now we will analyse the costs of SMCH algorithm and OCHO algorithm

\section{Cost of SMCH Algorithm}

Let us assume

$\mathrm{C}_{\mathrm{L}}=$ link establishment cost

$\mathrm{C}_{\mathrm{D}}=$ data transfer cost

$\mathrm{C}_{\mathrm{LA}}=$ link establishment cost for route augmentation

$\mathrm{C}_{\mathrm{LR}}=$ link establishment cost for reroute

$\mathrm{C}_{\mathrm{LA}}=$ data transfer cost for link augmentation

$\mathrm{C}_{\mathrm{DR}}=$ data transfer cost for reroute

$\mathrm{C}_{\mathrm{A}}=$ total handover cost using route augmentation scheme $=\mathrm{C}_{\mathrm{LA}}+\mathrm{C}_{\mathrm{DA}}$

$\mathrm{C}_{\mathrm{R}}=$ total handover cost for reroute $=\mathrm{C}_{\mathrm{LR}}+\mathrm{C}_{\mathrm{DR}}$

Generally $\mathrm{C}_{\mathrm{LA}}<\mathrm{C}_{\mathrm{LR}}$

As $\mathrm{SMCH}$ algorithm is done after handover so if the reroute is needed net cost for handover in rerouting scheme in $\mathrm{SMCH}$ algorithm is

$\mathrm{C}_{\mathrm{Net}}=\mathrm{C}_{\mathrm{LA}}+\mathrm{C}_{\mathrm{LR}}+\mathrm{C}_{\mathrm{DR}}$

So we see there is an extra cost $C_{\mathrm{LA}}$ in the above equation because link augmentation is done after handover and then the $\mathrm{SMCH}$ algorithm is performed. Though $\mathrm{C}_{\mathrm{LA}}<\mathrm{C}_{\mathrm{LR}}$ but we cannot ignore this cost as handover is very frequent. We will overcome this extra cost in our proposed OCHO algorithm. In OCHO algorithm this extra cost for rerouting is reduced. 


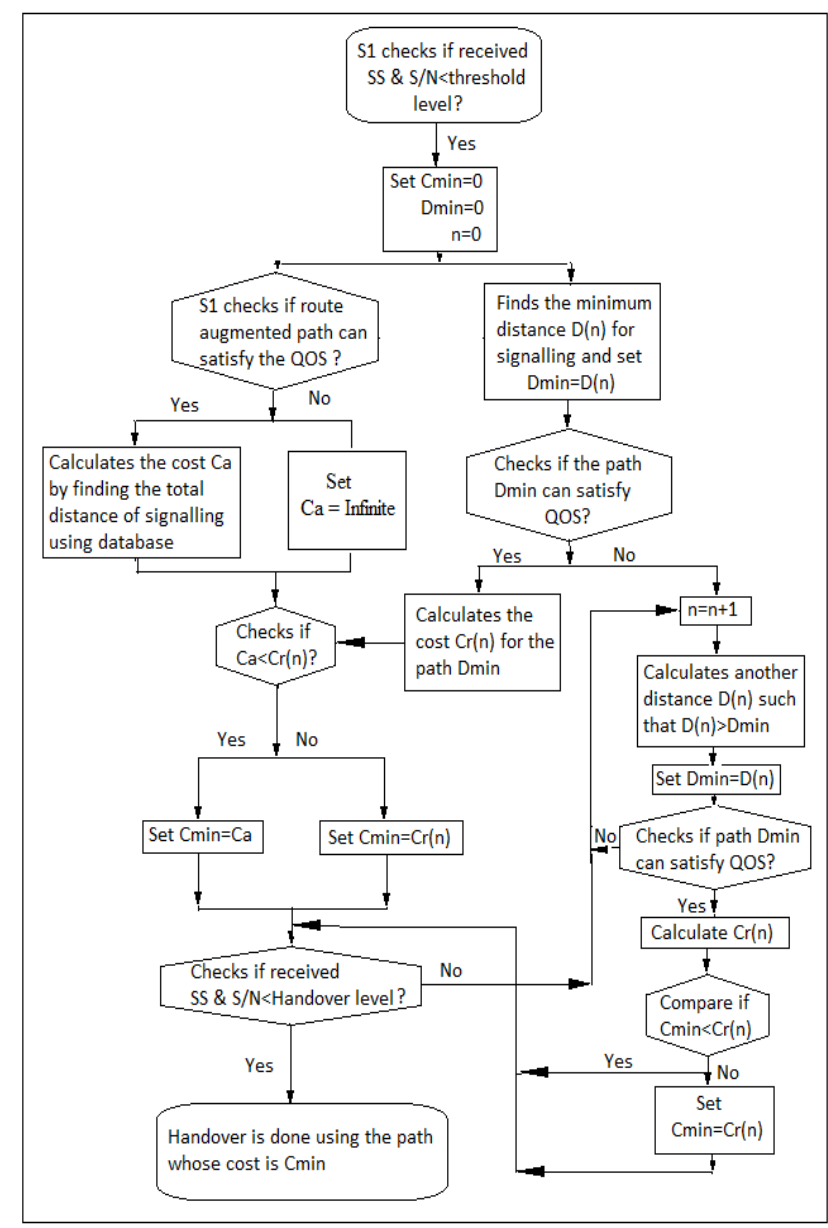

Fig.7 OCHO Algorithm

\section{Cost of OCHO Algorithm:}

The cost for case 1 is same as the cost of route augmentation of $\mathrm{SMCH}$ algorithm. If $\mathrm{C}_{1}$ denotes cost for case 1 then

$$
\mathrm{C}_{1}=\mathrm{C}_{\mathrm{LA}}+\mathrm{C}_{\mathrm{DA}}
$$

Now the cost of case 2 and case 3 are. If $\mathrm{C}_{2}$ and $\mathrm{C}_{3}$ denotes the cost of case 2 and case 3 respectively then

$$
\mathrm{C}_{2}=\mathrm{C}_{3}=\mathrm{C}_{\mathrm{LR}}+\mathrm{C}_{\mathrm{DR}}
$$

So we see that the extra cost of SMCH algorithm is rejected in $\mathrm{OCHO}$ algorithm

\section{Simulations results}

In order to evaluate the performance of OCHO algorithm we compare it to FHRP algorithm and SMCH algorithm. Each algorithm is evaluated by analysing the cost ratios, the average of different delay constraints and the percentage of path failing to satisfy the delay constraints . The simulation results were run in MATLAB 7.8 in a designed virtual environment.

In figure 8 we compare the handover cost of the OCHO algorithm with FHRP algorithm and SMCH algorithm. Let $\mathrm{OF}_{\mathrm{r}}$ represents the ratio of the handover costs between $\mathrm{OCHO}$ algorithm and FHRP algorithm and $\mathrm{OS}_{\mathrm{r}}$ denotes ratio of handover

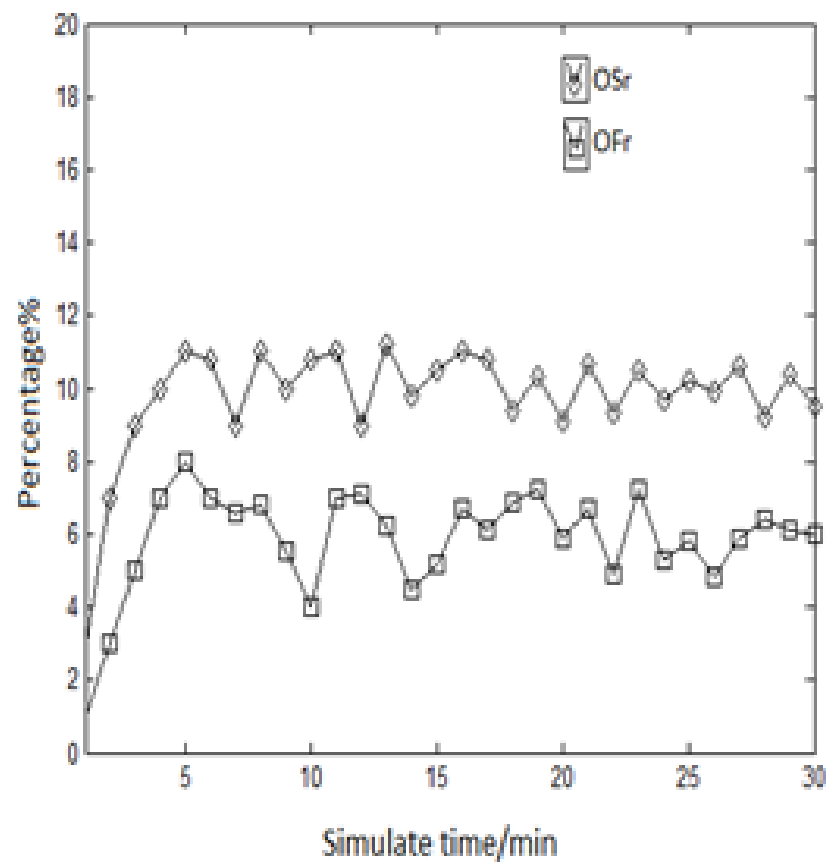

Fig.8 $\mathrm{OF}_{\mathrm{r}}$ and $\mathrm{OS}_{\mathrm{r}}$ results

costs of OCHO algorithm and SMCH algorithm.

$\mathrm{OF}_{\mathrm{r}}=$ (handover cost of $\mathrm{OCHO}$ algorithm)/ (handover cost of FHRP algorithm).

$\mathrm{OS}_{\mathrm{r}}=$ (handover cost of $\mathrm{OCHO}$ algorithm)/ (handover cost of SMCH algorithm).

As seen in figure $7 \mathrm{OCHO}$ algorithm has a lower handover cost than FHRP algorithm and SMCH algorithm. In the case of FHRP algorithm it can save nearly 6\% of network resources after handover and for $\mathrm{SMCH}$ algorithm the percentage is $10 \%$. OCHO algorithm is suitable where the no of handover is very frequent. The reason of low cost has been discussed in cost analysis section. 


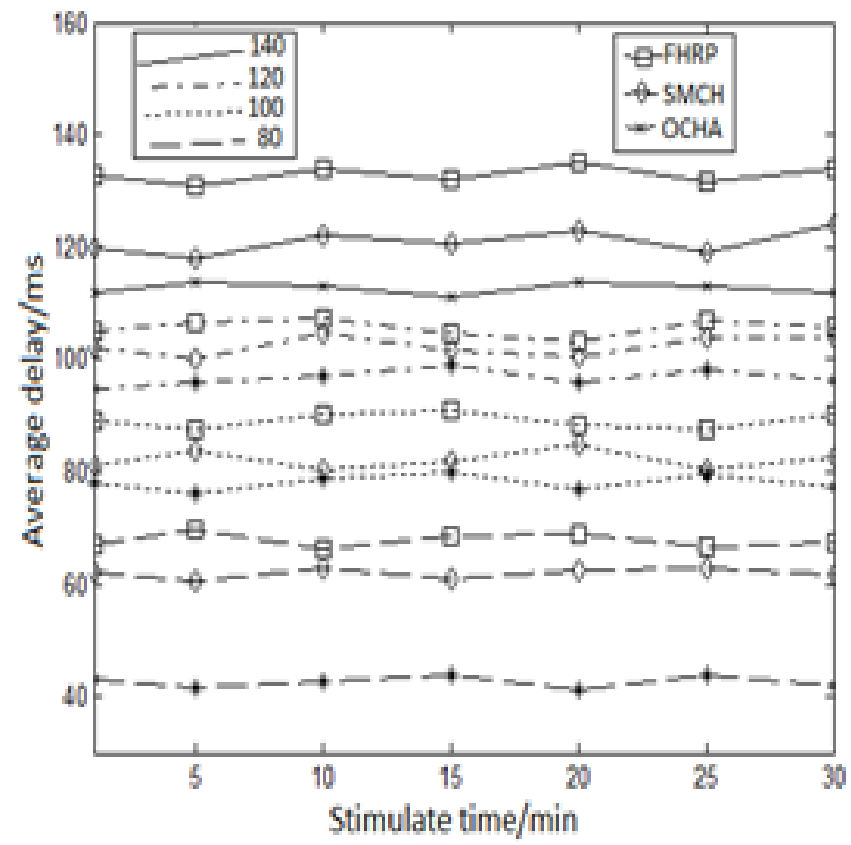

Fig.9 Averages of different delay constraints

In figure 9 we compare the QOS guarantee performance of OCHO algorithm with that of FHRP algorithm and SMCH algorithm in terms of delay constraints, which is very important parameter for mobile satellite networks.

In figure 7 we see that the delay performance of $\mathrm{OCHO}$ algorithm is better compared to FHRP algorithm and SMCH algorithm. In Fig. 8 we see the average delay of OCHO algorithm is less than FHRP and SMCH algorithm under same delay constraint. For example for the delay constraint 120 the average delay of FHRP algorithm is near about $132 \mathrm{~ms}$, for SMCH it is approximately $120 \mathrm{~ms}$ but for OCHO algorithm it is much lower i.e., $110 \mathrm{~ms}$ approx. The main reason for less delay is the future path is already known by OCHO algorithm. So the delay is only for establishing the connection which is much lower.

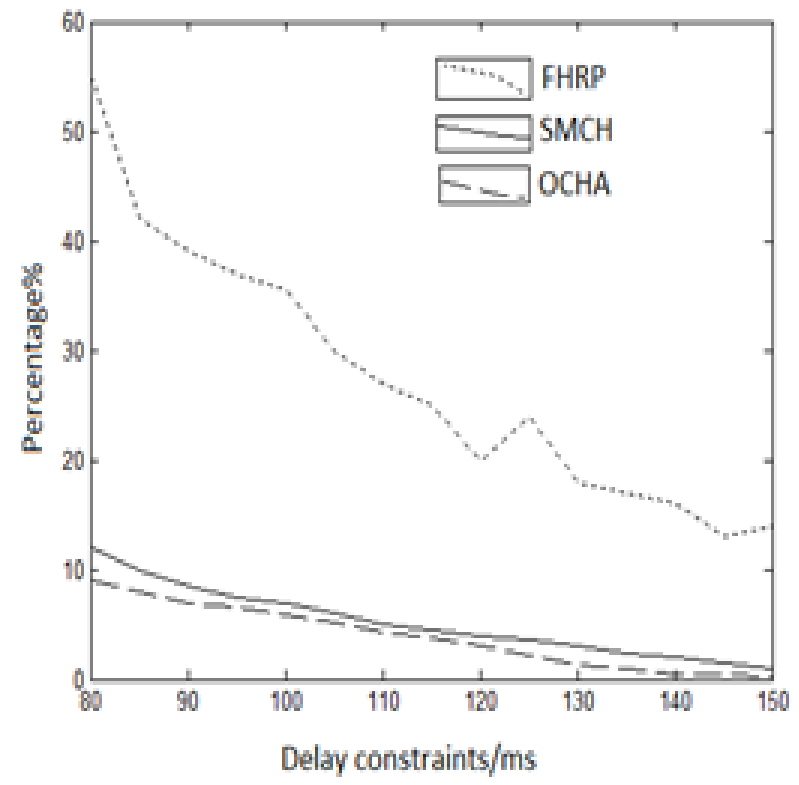

Fig.10 Percentage of path failing to satisfy the delay constraint
Fig.10 shows the percentage of path failing to satisfy the delay constraint for different algorithm. Suppose for delay constraint 100 nearly $35 \%$ of paths delay will exceed the delay constraint for FHRP algorithm and for SMCH algorithm it is nearly $9 \%$. But for OCHO algorithm nearly $6 \%$ of path will be rejected because the paths that satisfy the delay constraint for these calls cannot be found after handover.

\section{CONCLUSION:}

In this paper a new handover algorithm called Optimum Cost Handover Algorithm (OCHO)

Has been proposed which optimises the handover cost with the QOS parameters of handover service and optimises the result. It also reduces handover delay. Firstly we described different types of satellite and advantages of LEO satellites. Then we described the available handover schemes available mainly FHRP and SMCH algorithms and mentioned their demerits. Then we described our proposed OCHO algorithm and mentioned its advantages. The cost analysis of SMCH and $\mathrm{OCHO}$ algorithm is also shown after that. Then we create a virtual environment and we compared the FHRP, SMCH and OCHO algorithms and compared the costs of those algorithms and the average delay of these algorithms in terms of delay constrains. Results show that OCHO algorithm is superior to FHRP and SMCH algorithms.

\section{FUTURE WORK:}

In future we will be concentrating on finding proper distances of the three handover level so that it will optimise the OCHO algorithm's performance.

\section{REFERENCES}

[1] S. L. Kota, P. A. Leppanen, and K. Pahlavan, Broadband Satellite Communications For Internet Access, Kluwer Academic Publishers, 2004.

[2] C. Perkins, "IP mobility support," RFC 2002, IEFT, Oct. 1996, available at http://www.ietf.org/rfc/rfc2002.txt.

[3] P. K. Chowdhury, M. Atiquzzaman, W. Ivancic ,Handover Schemes in Satellite Networks: State-of-theart and future research directions IEEE communications surveys 4TH Quarter 2006, Vol. 8, NO. 4A.

[4] Soumya Das, Debabrata Sarddar, Dipsikha Ganguly, Suman Kumar Sikdar, Sougata Chakraborty and Kunal Hui. Article: Location Manager based Handover Method for LEO Satellite Networks. International Journal of Computer Applications44(12):43-49, April 2012. Published by Foundation of Computer Science, New York, USA.

[5] Debabrata Sarddar, Soumya Das, Dipsikha Ganguli, Kalyan Kumar Das, Sougata Chakraborty, Kunal Hui and Mrinal Kanti Naskar. Article: A New Method for Controlling Mobility Management Cost of PatHO-LEO satellite and Mobile IP Network. International Journal of Computer Applications 37(7):32-38, January 2012. Published by Foundation of Computer Science, New York, USA.

[6] Debabrata Sarddar, Dipsikha Ganguly, Soumya Das, Suman Kumar Sikdar, Sougata Chakraborty, Kunal Hui, Shabnam Bandyopadhyay, Kallyan Kumar Das and Mrinal Kanti Naskar. Article: Introduction of Handover Manager for Better Handover Latency and Handover 
Throughput during Leo Satellite Handover. International Journal of Computer Applications 42(15):47-52, March 2012. Published by Foundation of Computer Science, New York, USA.

[7] Debabrata Sarddar, Dipsikha Ganguly, Soumya Das, Suman Kumar Sikdar, Sougata Chakraborty, Kunal Hui, Shabnam Bandyopadhyay, Kallyan Kumar Das and Mrinal Kanti Naskar. Article: Area based Mobility Management by using GPS in LEO satellite Networks. International Journal of Computer Applications 42(13):41-43, March 2012. Published by Foundation of Computer Science, New York, USA.

[8] Debabrata Sarddar, Soumya Das, Dipsikha Ganguly, Suman Kumar Sikder, Sougata Chakraborty, Kunal Hui, Shabnam Bandyopadhyay, Kalyan Kumar Das and Mrinal Kanti Naskar. Article: Reducing the Mobile IP Binding Updates and the Packet Loss for the Repetitive IP Stations during a Short Period of Time. International Journal of Computer Applications 42(2):27-32, March 2012. Published by Foundation of Computer Science, New York, USA.

[9] Debabrata Sarddar, Soumya Das, Dipsikha Ganguly, Suman Kumar Sikder, Sougata Chakraborty, Kunal Hui, Shabnam Bandyopadhyay, Kalyan Kumar Das and Mrinal Kanti Naskar. Article: A Time based Mobility Management Method for Leo Satellite Networks. International Journal of Computer Applications 42(2):33-40, March 2012. Published by Foundation of Computer Science, New York, USA

[10] A. Jamalipour, "Satellites in IP networks," in Wiley Encyclopaedia of Telecommunications, vol. 4, Wiley, 2002, pp. 2111-2122.

[11] P. Bhagwat, C. Perkins, and S. Tripathi, "Network layer mobility: An architectureand survey," IEEE Pers. Commun., vol. 3, no. 3, pp. 54-64, June1996.

[12] E. Papapetrou and F.-N. Pavlidou, "QoS handover management in LEO/MEO satellite systems," Wireless Personal Communications, vol. 24, no. 2, pp. 189-204, February 2003.

[13] P. K. Chowdhury, M. Atiquzzaman, W. Ivancic ,Handover Schemes in Satellite Networks: State-of-theart and future research directions IEEE communications surveys 4TH Quarter 2006, Vol. 8, NO. 4A.

[14] A. Ganz, Y. Gong, and B. Li, "Performance study of low Earth orbit satellite systems," IEEE Transactions on Communications, vol. 42, no. 2/3/4, pp. 1866-1871, February - April 1994

[15] Del Re, E., Fantacci, R., and Giambene, G. "Handover queuing strategies with dynamic and fixed channel allocation techniques in low earth orbit mobile satellite systems",. IEEE Trans. Commun, Vol. 47, no.1, pp. 89101. Jan. 1999.

[16] Uzunalioglu H, Akyildiz I F, Yesha Y, et al. Footprint handover rerouting protocol for LEO satellite networks. ACM Wireless Networks 1999; 5(5): 327-337.

[17] S. Kalyanasundaram, E.K.P Chong, and N.B. Shroff, "An efficient scheme to reduce handoff dropping in LEO satellite systems," Wireless Networks, vol. 7, no. 1, pp. 75-85, January 2001.
[18] Wang L, Zhang N T. Dynamic probability path optimization strategy for satellite handoff in LEO networks. Journal of China Institute of Communications 2002; 23(9): 8-15. [in Chinese]

[19] Zhang Tao, Zhang Jun , "A Minimum Cost Handover Algorithm for Mobile Satellite Networks", Chinese Journal of Aeronautics 21(2008) 269-274, Received 6 November 2007; accept at 6 March 2008.

[20] Debabrata Sarddar, Soumya Das, Dipsikha Ganguli, Sougata Chakraborty, Kunal Hui, Kalyan Kumar Das and Mrinal Kanti Naskar. Article: A New Method for Fast and Low Cost Handover in Leo Satellites. International Journal of Computer Applications 37(7):3945, January 2012. Published by Foundation of Computer Science, New York, USA

[21] Debabrata Sarddar, Dipsikha Ganguly, Soumya Das, Suman Kumar Sikdar, Sougata Chakraborty, Kunal Hui, Shabnam Bandyopadhyay, Kalyan Kumar Das and Sujoy Palit. Article: Cost Analysis of Mobile IP for the Repetitive IP Stations during a Short Period of Time. International Journal of Computer Applications 45(19):7-12, May 2012. Published by Foundation of Computer Science, New York, USA.

[22] Debabrata Sarddar, Dipsikha Ganguly, Soumya Das, Suman Kumar Sikdar, Sougata Chakraborty, Kunal Hui, Shabnam Bandyopadhyay, Kalyan Kumar Das and Sujoy Palit. Article: Cost Analysis of Location Manager based Handover Method for LEO Satellite Networks. International Journal of Computer Applications 45(19):1-6, May 2012. Published by Foundation of Computer Science, New York, USA

[23] Debabrata Sarddar, Dipsikha Ganguly, Soumya Das, Suman Kumar Sikdar, Sougata Chakraborty, Kunal Hui, Shabnam Bandyopadhyay, Kalyan Kumar Das and Sujoy Palit. Article: Handoff Latency Minimization by using Access Point by GPS using Selective scanning. International Journal of Computer Applications 45(19):13-19, May 2012. Published by Foundation of Computer Science, New York, USA.

\section{AUTHORS}

Debabrata Sarddar is currently pursuing his $\mathrm{PhD}$ at Jadavpur University. He completed his M.Tech in Computer Science \& Engineering from DAVV, Indore in 2006, and his B.Tech in Computer Science \& Engineering from Regional Engineering College, Durgapur in 2001. His research interest includes wireless and mobile communication.

Soumya Das, son of Mr. Subrata Das and Mrs. Swapna Das, currently pursuing his B.Tech in Electronics \& Communication Engg. at Bengal Institute of Technology under West Bengal University of Technology. His research interest includes mobile communication \& satellite communication.

Dipsikha Ganguly, currently pursuing B.Tech in Electronics \& Communication engineering at B.P.Poddar Institute of Management \& Technology under West Bengal University of Technology. Her research interest includes wireless communication \& satellite communication. 
Suman Kumar Sikdar completed his B.Tech in CSE from Murshidabad College of Engineering \& Technology and M.Tech in Computer Science \& Engineering from Jadavpur University in 2011. His research interest includes wireless sensor networks and wireless communication systems.

Sougata Chakraborty is presently an employee of IBM (Kolkata). He has completed his B.Tech in IT from Murshidabad College of Engineering and Technology and M.Tech in Computer Science \& Engineering from Jadavpur University in 2011. His research interest includes wireless sensor networks and wireless communication systems.
Kunal Hui is presently an employee of Cognizant (Kolkata). $\mathrm{He}$ has completed his B.Tech in Computer Science \& Engineering from Murshidabad College of Engineering and Technology (2004-2008) and M.Tech in Computer Science \& Engineering from Haldia Institute of Technology (20092011). His research interest includes wireless sensor networks and wireless communication systems.

Sujoy Palit completed his M. Tech degree in Computer Science and Engineering from West Bengal University of Technology in 2005. His research interest includes wireless sensor networks and wireless communication systems. 